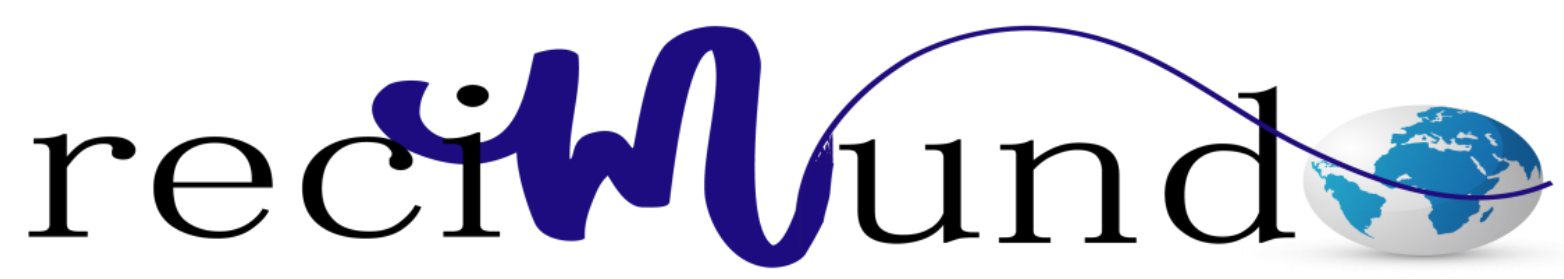

Revista Cientifica Mundo de la Investigación y el Conocimiento

Priscila Fernanda Vintimilla Enderica ${ }^{\text {a }}$; Yuri Olivo Giler Mendoza ${ }^{\text {b; Katherine }}$ Elizabeth Motoche Apolo ${ }^{c}$; Jenny Jazmín Ortega Flores ${ }^{d}$

Diabetes Mellitus Tipo 2: Incidencias, Complicaciones y Tratamientos Actuales

Diabetes Mellitus Type 2: Incidents, Complications and Current Treatments

Revista Científica Mundo de la Investigación y el Conocimiento. Vol. 3 núm.1, enero, ISSN: 2588-073X, 2019, pp. 26-37

DOI: 10.26820/recimundo/3.(1).enero.2019.26-37

URL: http://www.recimundo.com/index.php/es/article/view/355

Editorial Saberes del Conocimiento

Recibido: 20/11/2018

Aceptado: 05/01/2019

Publicado: $31 / 01 / 2019$

Correspondencia: director@ recimundo.com
a. Médico de la Universidad de Guayaquil.
b. Magister en Gerencia de Servicios de Salud; Especialista en Gestión de Servicios de Salud; Diploma Superior en Gestión de Desarrollo de los Servicios de Salud; Medico.
c. Médico de la Universidad de Guayaquil.
d. Médico de la Universidad de Guayaquil. 


\section{Diabetes Mellitus Tipo 2: Incidencias, Complicaciones y Tratamientos \\ Actuales}

Vol. 3, núm. 1., (2019)

Priscila Fernanda Vintimilla Enderica; Yuri Olivo Giler Mendoza; Katherine Elizabeth Motoche

Apolo; Jenny Jazmín Ortega Flores

\section{RESUMEN}

En la siguiente investigación se trató como tema la diabetes mellitus tipo 2, además de sus incidencias, complicaciones y tratamientos actuales. Se debe especificar como primer punto que el término diabetes mellitus (DM) define alteraciones metabólicas de múltiples etiologías caracterizadas por hiperglucemia crónica y trastornos en el metabolismo de los hidratos de carbono, las grasas y las proteínas, resultado de defectos en la secreción de insulina, en la acción de la misma o en ambas. La diabetes mellitus tipo 2 se considera una de las enfermedades crónicas con mayor impacto en la calidad de vida de la población mundial y constituye un verdadero problema de salud. Se estima que cerca de 4 millones de muertes al año están relacionadas directamente con esta afección, avalado por los cambios en los estilos de vida de la población, asociados al sedentarismo, la obesidad, la hipertensión arterial y otros factores de riesgo cardiovasculares. Se obtuvo como resultados que la DM tipo 2 es una enfermedad crónica de prevalencia creciente. La magnitud del problema, en lo que hace referencia a los gastos directos e indirectos, nos tiene que hacer ser muy cautos para decidir cuál es el mejor tratamiento para los pacientes. Como principal conclusión se aseveró que la modificación del estilo de vida es la piedra angular en la prevención y el control metabólico del paciente diabético tipo 2, sin embargo, existe un amplio grupo de medicamentos con características farmacológicas variadas que los hacen ser muy usados en la práctica diaria, pues tienen la finalidad de disminuir la toxicidad por altos niveles de glucosa en sangre.

Palabras claves: Diabetes; Metabolismo; Hiperglucemia; Obesidad y Enfermedades Crónicas. 


\section{Diabetes Mellitus Tipo 2: Incidencias, Complicaciones y Tratamientos Actuales}

Vol. 3, núm. 1., (2019)

Priscila Fernanda Vintimilla Enderica; Yuri Olivo Giler Mendoza; Katherine Elizabeth Motoche Apolo; Jenny Jazmín Ortega Flores

\section{ABSTRACT}

In the following research, type 2 diabetes mellitus was treated as a topic, as well as its incidences, complications and current treatments. It should be specified as the first point that the term diabetes mellitus (DM) defines metabolic alterations of multiple etiologies characterized by chronic hyperglycemia and disorders in the metabolism of carbohydrates, fats and proteins, resulting from defects in insulin secretion, in the action of the same or in both. Diabetes mellitus type 2 is considered one of the chronic diseases with greater impact on the quality of life of the world population and constitutes a real health problem. It is estimated that about 4 million deaths per year are directly related to this condition, supported by changes in the lifestyles of the population, associated with sedentary lifestyle, obesity, high blood pressure and other cardiovascular risk factors. It was obtained as results that type 2 DM is a chronic disease of increasing prevalence. The magnitude of the problem, in what refers to direct and indirect costs, has to make us very cautious to decide what the best treatment for patients is. The main conclusion was that lifestyle modification is the cornerstone in the prevention and metabolic control of type 2 diabetic patients; however, there is a wide group of drugs with varied pharmacological characteristics that make them widely used in the daily practice, because they have the purpose of reducing toxicity by high blood glucose levels.

Key words: Diabetes, Metabolism, Hyperglycemia, Obesity and Chronic Diseases. 


\section{Diabetes Mellitus Tipo 2: Incidencias, Complicaciones y Tratamientos \\ Actuales}

Vol. 3, núm. 1., (2019)

Priscila Fernanda Vintimilla Enderica; Yuri Olivo Giler Mendoza; Katherine Elizabeth Motoche Apolo; Jenny Jazmín Ortega Flores

\section{Introducción.}

El término diabetes mellitus (DM) define alteraciones metabólicas de múltiples etiologías caracterizadas por hiperglucemia crónica y trastornos en el metabolismo de los hidratos de carbono, las grasas y las proteínas, resultado de defectos en la secreción de insulina, en la acción de la misma o en ambas. La DM puede presentarse con síntomas característicos, como sed, poliuria, visión borrosa y pérdida de peso. Frecuentemente, los síntomas no son graves o no se aprecian. Por ello, la hiperglucemia puede provocar cambios funcionales y patológicos durante largo tiempo antes del diagnóstico. (Sistema Nacional de Salud, 2008)

La diabetes mellitus tipo 2 se considera una de las enfermedades crónicas con mayor impacto en la calidad de vida de la población mundial y constituye un verdadero problema de salud; pertenece al grupo de las enfermedades que producen invalidez física por sus variadas complicaciones multiorgánicas, con un incremento indudable en la morbilidad y mortalidad en los últimos años, independientemente de las circunstancias sociales, culturales y económicas de los países. (Reyes, Pérez, Figueredo, Estupiñan, \& Jiménez, 2016)

En el mundo existen 170 millones de personas afectadas por diabetes mellitus que se duplicarán para el 2030. Para ese año, en América Latina se calcula un incremento de $148 \%$ de los pacientes con diabetes. En México se estima que de 6.8 millones de afectados se pasará a 11.9 millones, con un incremento de $175 \%$. El número de casos nuevos de diabetes en niños y adolescentes mexicanos se triplicó entre 1990 y 2007, particularmente entre los mayores de 25 años; el grupo más afectado fue el de 15 a 19 años a partir del año 2000 y el número de casos en 


\section{Diabetes Mellitus Tipo 2: Incidencias, Complicaciones y Tratamientos Actuales}

Vol. 3, núm. 1., (2019)

Priscila Fernanda Vintimilla Enderica; Yuri Olivo Giler Mendoza; Katherine Elizabeth Motoche Apolo; Jenny Jazmín Ortega Flores

2007 se multiplicó casi por cinco: pasó de 411 a 1770 casos. (Gil, Sil, Domínguez, Torres, \&

Medina, 2013)

Se estima que cerca de 4 millones de muertes al año están relacionadas directamente con esta afección (lo que equivale a una de cada 20 muertes, 8700 muertes cada día y 6 cada minuto) avalado por los cambios en los estilos de vida de la población, asociados al sedentarismo, la obesidad, la hipertensión arterial y otros factores de riesgo cardiovasculares. Por esas razones, ocupa la cuarta causa de muerte en todo el universo (Reyes, Pérez, Figueredo, Estupiñan, \& Jiménez, 2016)

La hiperglucemia crónica se asocia con lesiones a largo plazo en diversos órganos, particularmente ojos, riñón, nervios, vasos sanguíneos y corazón. Diversos procesos patogénicos están involucrados en el desarrollo de DM, aunque la gran mayoría de los casos pueden incluirse en dos categorías. En la primera de ellas, diabetes mellitus de tipo 1 (DM1), la causa es una deficiencia absoluta en la secreción de insulina, a menudo con evidencia de destrucción autoinmune de las células $\beta$ pancreáticas. En la segunda categoría, mucho más prevalente, diabetes mellitus de tipo 2 (DM2), la causa es una combinación de resistencia a la acción de la insulina (generalmente asociada a obesidad) y una inadecuada respuesta secretora compensatoria. (Almaro, Botella, \& Simal, 2000)

Las complicaciones crónicas de la DM incluyen el progresivo desarrollo de retinopatía, con potencial ceguera; nefropatía que puede llevar al fallo renal; neuropatía periférica con riesgo de úlceras plantares, amputación o pie de Charcot; determinadas infecciones; alteraciones odontológicas, neuropatía autonómica; y enfermedades cardiovasculares, como cardiopatía 


\section{Diabetes Mellitus Tipo 2: Incidencias, Complicaciones y Tratamientos \\ Actuales}

Vol. 3, núm. 1., (2019)

Priscila Fernanda Vintimilla Enderica; Yuri Olivo Giler Mendoza; Katherine Elizabeth Motoche Apolo; Jenny Jazmín Ortega Flores

isquémica, accidentes cerebrovasculares o arteriopatía periférica (Sistema Nacional de Salud, 2008)

La atención eficiente de los pacientes diabéticos implica un trabajo coordinado y multidisciplinar con la participación de la atención primaria y especializada. La diabetes mellitus tipo 2 es una enfermedad en la que constantemente se están produciendo avances, tanto en el diagnóstico como en lo que se refiere a su manejo y tratamiento. Los cambios en los criterios diagnósticos, la comercialización de nuevos fármacos para el control glucémico y la continua publicación de nuevos estudios sobre la eficacia del control de los factores de riesgo cardiovascular necesitan ser evaluados y, según el caso, incorporados a la práctica clínica por los profesionales responsables de la atención a los pacientes diabéticos. (Sistema Nacional de Salud, 2008)

La diabetes mellitus tipo 2 (DM2) es una enfermedad crónica de prevalencia creciente asociada a la obesidad, el sedentarismo y el envejecimiento de la población. La magnitud del problema obliga a ser muy cuidadosos en el momento de decidir el mejor tratamiento cuando no se logran objetivos de control todo y las medidas higienicodietéticas y la metformina. El tratamiento de esta enfermedad, una vez establecida, está orientado a evitar el desarrollo de descompensaciones agudas y de las complicaciones crónicas que determinan la morbilidad y la mortalidad. La base de este tratamiento son las medidas higienicodietéticas y la metformina. Durante muchos años, la única alternativa a la metformina fueron las sulfonilureas (SU) y la insulina. Posteriormente, se incorporaron los inhibidores de las glucosidasas y las tiazolidindionas (TZD). A lo largo de los últimos años, pero, se han desarrollado otras muchas 


\section{Diabetes Mellitus Tipo 2: Incidencias, Complicaciones y Tratamientos Actuales}

Vol. 3, núm. 1., (2019)

Priscila Fernanda Vintimilla Enderica; Yuri Olivo Giler Mendoza; Katherine Elizabeth Motoche Apolo; Jenny Jazmín Ortega Flores

dianas terapéuticas diferentes que han supuesto la aparición de varios grupos farmacológicos.

(Fernandez \& Ricart, 2015)

\section{Materiales y Métodos.}

Para llevar adelante el siguiente trabajo académico se recurrió al método de la investigación documental. Esta herramienta se fundamenta en la recolección de documentos, categorizarlos, analizarlos; para después poder presentar un resultado coherente. El objetivo de esta metodología es poder aportar nuevos conocimientos. (Reyna, 1995)

También se puede decir que esta estrategia se basa en la observación y reflexión sistemática de realidades teóricas y empíricas, en la que usa diversos tipos de documentos que sirven para indagar, interpretar, exponer cifras e informaciones referentes al tema de ciencia que se trabaje. Todo utilizando instrumentos que tienen como meta conseguir resultados que pueden ser la plataforma para otra exploración científica y la construcción de nuevos conocimientos. (Bravo, 1999)

De igual forma, se puede enumerar en los siguientes pasos: realizar un proceso de abstracción científica, generalizando sobre la base de lo fundamental; utilizar los procedimientos lógicos y mentales de toda investigación; tales como: análisis, síntesis, deducción, inducción, entre otros; y se debe a una recopilación adecuada de datos, que permiten redescubrir hechos, sugerir problemas, ubicar hacia otras fuentes de investigación, orientar formas para elaborar instrumentos de investigación y elaborar hipótesis (Rodríguez, 2013)

Dicho estudio se respaldó con una investigación bibliográfica permite, entre otras cosas, evitar iniciar exploraciones anteriormente realizadas, obtener conocimiento de experimentos 


\section{Diabetes Mellitus Tipo 2: Incidencias, Complicaciones y Tratamientos \\ Actuales}

Vol. 3, núm. 1., (2019)

Priscila Fernanda Vintimilla Enderica; Yuri Olivo Giler Mendoza; Katherine Elizabeth Motoche Apolo; Jenny Jazmín Ortega Flores

previos para repetirlos en caso de ser necesario, buscar datos sugerentes, culminar investigaciones interrumpidas o incompletas, seleccionar los materiales y documentos para un marco teórico. (López, Vaquero, \& Rául, 2001)

Con lo expuestos se puede asegurar que en el presente trabajo de investigación se utilizó como metodología una revisión bibliográfica documental no experimental, puesto que realizó una revisión y análisis de diferentes artículos médicos y de opinión referentes a la incidencia de ladiabetes mellitus tipo 2, para poder abordar de la mejor manera.

\section{Resultados.}

La hiperglucemia persistente es el fenómeno central en todas las formas de DM. El tratamiento debe estar encaminado a descender los niveles de glucemia a valores próximos a la normalidad siempre que sea posible. Con ello perseguimos: 1. Evitar descompensaciones agudas, cetoacidosis o síndrome hiperosmolar. 2. Aliviar los síntomas cardinales (poliuria/ polidipsia/ astenia/ pérdida de peso con polifagia). 3. Minimizar el riesgo de desarrollo o progresión de retinopatía, nefropatía y/o neuropatía diabética. 4. Evitar las hipoglucemias 5. Mejorar el perfil lipídico de los pacientes. 6. Disminuir la mortalidad. (Almaro, Botella, \& Simal, 2000)

La DM tipo 2 es una enfermedad crónica de prevalencia creciente. La magnitud del problema, en lo que hace referencia a los gastos directos e indirectos, nos tiene que hacer ser muy cautos para decidir cuál es el mejor tratamiento para nuestros pacientes. Los nuevos medicamentos descritos, suponen alternativas de eficacia discreta en relación a la mejora del control glucémico medido mediante los niveles de HbA1c en comparación con fármacos más 


\section{Diabetes Mellitus Tipo 2: Incidencias, Complicaciones y Tratamientos Actuales}

Vol. 3, núm. 1., (2019)

Priscila Fernanda Vintimilla Enderica; Yuri Olivo Giler Mendoza; Katherine Elizabeth Motoche Apolo; Jenny Jazmín Ortega Flores

antiguos como por ejemplo las SU, la insulina o las TZD, a los que superan en el aspecto de la ganancia ponderal y el desarrollo de hipoglucemias. (Fernandez \& Ricart, 2015)

Asimismo, es importante destacar que además del tratamiento farmacológico se debe tener en cuenta los siguientes factores : El tratamiento preventivo: múltiples ensayos clínicos aleatorizados recientes demuestran que personas con alto riesgo de desarrollar diabetes mellitus tipo 2 (pacientes con antecedentes de diabetes mellitus en familiares primer grado, obesidad, hipertensión arterial, personas mayores de 45 años de edad, mujeres con hijos macrosómicos, con síndrome de ovario poliquístico, alteración de la glucosa en ayunas, así como, tolerancia a la glucosa alterada), pueden retrasar su aparición, a través de programas bien estructurados para modificar estilos de vida. En el $58 \%$ de estos pacientes, se logra reducir el debut de esta enfermedad durante 3 años, con el uso de los siguientes fármacos: metformina, acarbosa, repaglinida y roziglitazona que son también efectivos y el tratamiento no farmacológico: el tratamiento no farmacológico (modificación del estilo de vida y en especial la reducción del peso corporal en el paciente sobrepeso) es el único tratamiento integral capaz de controlar simultáneamente la mayoría de los problemas metabólicos de las personas con diabetes, incluyendo la hiperglicemia, la resistencia a la insulina, la dislipoproteinemia y la hipertensión arterial. Además, comprende el plan de educación terapéutica, alimentación, ejercicios físicos y hábitos saludables. (Reyes, Pérez, Figueredo, Estupiñan, \& Jiménez, 2016)

\section{Conclusiones.}

La modificación del estilo de vida es la piedra angular en la prevención y el control metabólico del paciente diabético tipo 2, sin embargo, existe un amplio grupo de medicamentos 


\section{Diabetes Mellitus Tipo 2: Incidencias, Complicaciones y Tratamientos \\ Actuales}

Vol. 3, núm. 1., (2019)

Priscila Fernanda Vintimilla Enderica; Yuri Olivo Giler Mendoza; Katherine Elizabeth Motoche Apolo; Jenny Jazmín Ortega Flores

con características farmacológicas variadas que los hacen ser muy usados en la práctica diaria, pues tienen la finalidad de disminuir la toxicidad por altos niveles de glucosa en sangre. (Reyes, Pérez, Figueredo, Estupiñan, \& Jiménez, 2016)

En el manejo terapéutico de la DM2 no hay que olvidar que para la prevención o retraso de las complicaciones micro y, sobre todo, macrovasculares es fundamental el control precoz de otros factores de riesgo muy frecuentemente acompañantes como son la hipertensión arterial, la dislipemia y el hábito tabáquico. (Almaro, Botella, \& Simal, 2000)

La prevalencia de la diabetes aumenta con la edad. Es inferior al $10 \%$ en personas menores de 60 años y entre el 10\%-20\% entre los 60-79 años de edad. Existe una mayor prevalencia en varones entre 30 y 69 años y en las mujeres mayores de 70 años. (Sistema Nacional de Salud, 2008)

\section{Recomendaciones.}

Una intervención educativa que cambie el estilo de vida con dieta hipocalórica baja en grasas y ejercicio físico (al menos durante 150 minutos a la semana) es más efectiva que la metformina en la prevención de la diabetes. El proceso educativo se debe establecer permanentemente.

Los médicos familiares deben realizar una historia clínica completa en los pacientes mayores de 16 años que les permita identificar los factores de riesgo para diabetes mellitus Deberá realizarse detección sistemática de diabetes tipo 2 en adultos asintomáticos de cualquier edad sobrepeso u obesidad y otros factores de riesgo, y en los sujetos que no tienen factores de riesgo, a la edad de 45 años. (Gil, Sil, Domínguez, Torres, \& Medina, 2013) 


\section{Diabetes Mellitus Tipo 2: Incidencias, Complicaciones y Tratamientos Actuales}

Vol. 3, núm. 1., (2019)

Priscila Fernanda Vintimilla Enderica; Yuri Olivo Giler Mendoza; Katherine Elizabeth Motoche Apolo; Jenny Jazmín Ortega Flores

El surgimiento de nuevas terapias ayuda a mejorar la calidad de vida de los enfermos, por

lo que su conocimiento brinda al médico múltiples alternativas en el manejo del paciente diabético, tanto en la atención primaria, como en la secundaria. (Reyes, Pérez, Figueredo, Estupiñan, \& Jiménez, 2016)

\section{Referencias Bibliográficas}

Almaro, J., Botella, F., \& Simal, A. (2000). Tratamiento de la diabetes mellitus. Información terapéutica del Sistema Nacional de Salud, 33-43.

Bravo, S. (1999). Tesis doctorales y trabajos de investigación científica: metodología general de su eleboración y documentación. Madrid: Parainfo.

Fernandez, M., \& Ricart, W. (2015). Nuevos tratamientos farmacológicos para la diabetes mellitus tipo 2: Los agentes incretínicos y agentes glucosúricos. CatSalut, 1-9.

Gil, L., Sil, M., Domínguez, E., Torres, L., \& Medina, J. (2013). Guía de práctica clínica: Diagnóstico y tratamiento de la diabetes mellitus tipo 2. Revista de instrumentos clínicos México, 103-119.

López, L., Vaquero, J., \& Rául, J. (2001). Manual de Investigación bibliográfica y documental. Madrid: Pirámide.

Martínez, S. (2002). Guía de apuntes básicos para el docente de la materia técnicas de investigación. Oaxaca: Grupo emergente de investigación Oaxaca.

Parraguez, S., Chunga, G., Flores, M., \& Romero, R. (2017). El estudio y la invstigacion documental: estrategias metodologicas y herramientas tic . Chiclayo: EMDOCOSEGE S.A.

Reyes, F., Pérez, M., Figueredo, E., Estupiñan, M., \& Jiménez, Y. (2016). Tratamiento actual de la diabetes mellitus tipo 2. Correo científico médico , 98-121.

Reyna, S. (1995). Manual de redacción e investigación documental. Mexico DF: Editorial Trillas.

Rodríguez, S. (2013). Acerca de la investigación bibliógrafica y documental. Obtenido de Guia de Tesis: http://guiadetesis. wordpress. com/2013/08/19/acerca-de-la-investigacionbibliografica-ydocumental 


\section{Diabetes Mellitus Tipo 2: Incidencias, Complicaciones y Tratamientos}

Actuales

Vol. 3, núm. 1., (2019)

Priscila Fernanda Vintimilla Enderica; Yuri Olivo Giler Mendoza; Katherine Elizabeth Motoche Apolo; Jenny Jazmín Ortega Flores

Sampieri, R. (1998). Metodologia de la investigacion. México DF: Interamericana editores. Sistema Nacional de Salud. (2008). Guía de Práctica Clínica sobre Diabetes tipo 2. Vitoria, Gateiz, España. 